\title{
SYNTHESIS OF NONLINEAR CONTROL STRATEGIES FROM FUZZY LOGIC CONTROL ALGORITHMS
}

\author{
Reza Langari \\ Department of Mechanical Engineering \\ Texas A\&M University \\ College Station, TX 7783-3123
}

\begin{abstract}
Fuzzy control has been recognized as an alternative to conventional control techniques in situations where the plant model is not sufficiently well known to warrant the application of conventional control techniques. Precisely what fuzzy control does and how it does what it does is not quite clear, however. This paper deals with this important issue and in particular shows how a given fuzzy control scheme can resolve into a nonlinear control law and that in those situations the success of fuzzy control hinges on its ability to compensate for nonlinearities in plant dynamics.
\end{abstract}

\section{INTRODUCTION}

Fuzzy logic control has been recognized as an altemative to conventional control techniques(primarily PID, or switching type control) for application in industrial process control and manufacturing automation(Sugeno 1985). More often than not, however, empirical observation provides the only means to a comparative study of performance of fuzzy controllers in relation to their conventional counterparts. While this fact is recognized and even appreciated by practitioners in the process control area, precisely what a fuzzy controller does, that is from an analytical standpoint, and how it does what it does is still of interest.

In order to investigate this issue, we will consider the notion of parametrized fuzzy sets and discuss its implication in analysis of fuzzy control algorithms. This idea, it turns out(Langari and Tomizuka 1990, Langari 1990, Langari 1992) gives rise to a framework for analysis and synthesis of nonlinear control strategies that emerge quite naturally from an initial statement of a given control strategy as a fuzzy linguistic control algorithm.

In this article, we will use this framework to explain how a given fuzzy control strategy deals with process nonlinearities that conventional controllers, for instance PID, generally do not. In particular, we apply this framework to the problem of control synthesis in a typical situation where asymmetric response characteristics of the process precludes, or severely encumbers the application of conventional(linear) control theory. We further show how in this situation an appropriately designed fuzzy controller overcomes this difficulty and by in effect compensating for the underlying nonlinearities produces superior behavior.

We start with an overview of fuzzy control

\section{FUZZY CONTROL SYSTEMS}

The typical architecture of a fuzzy control systems in shown in Figure 1. As a rule based control strategy, fuzzy linguistic control is based on explicit representation of knowledge of operation of the process as condition action rules of the form

$$
R_{J, S}: \text { if } e(t) \text { is } A_{J} \text { and } d e(t) \text { is } B, \text { then } u(t) \text { is } C_{J,}
$$

where $e(t)$ denotes the instantaneous value of the

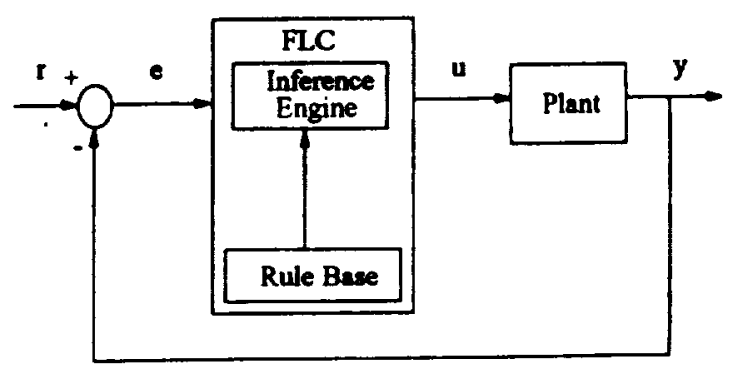

Figure 1 Architecture of a Fuzzy Logic Control System 
process error at time $t$ and $\operatorname{de}(t)$ is short for $\mathscr{D}(e ; t)$, which stands for $\frac{d e}{d t}$ or $\int e d \tau$. Further, $\tilde{A}_{j}$, $\tilde{B}_{1}$, and $\bar{C}_{j,}$ belong to collections $\dot{\bar{A}}, \bar{s}_{\bar{B}}$, and $\bar{B}$ of fuzzy subsets defined over the domains of definition of the relevant variables, that is, $E, D E$, and $U$ respectively and $R_{j, l}$ denotes the $j, l^{\text {th }}$ rule in the rule set $\mathbf{R}$. In particular $R_{j \ell}$ may be
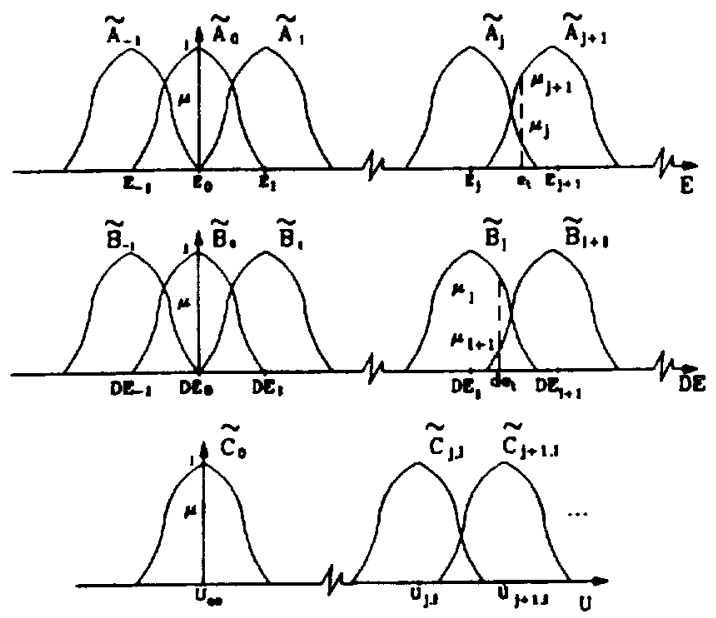

Figure 2. Fuzzy partitioning of the domains of definition.

be viewed as associating elements $\tilde{A}$, of $\dot{A}$ and $\tilde{B}_{t}$ of $\overline{98}$ with element $\tilde{C}_{, l}$ of $\overline{8}^{-}$, thereby forming a fuzzy relation ${ }^{1} \tilde{R}_{j, l}$ over the Cartesian product space, $E \times D E \times U$. From this standpoint, the given fuzzy control algorithm in effect amounts to a disjunction of such associations, as in $\tilde{R}_{\mathscr{R}}=y_{j, l} \tilde{R}_{j, \lambda}$, which Mamdani and Assilian(1975) refer to as the fuzzy relation matrix.

\section{Control Computation}

Suppose, at some instance $t$, as shown in Figure 2, the error $e(t)$ has positive grades of membership, $\mu_{\lambda_{j}}(e(t))$ and $\mu_{i_{j+1}}(e(t))$ to some pair $\tilde{A}_{j}$ and $\tilde{A}_{j+1}$ in $\tilde{A}$. Similarly, suppose $d e(t)$ belongs to some pair $\tilde{B}_{l}$ and $\tilde{B}_{l+1}$ in $\tilde{B}^{-}$. At this instant, the following control rules apply

${ }^{1}$ Note that the distinction in the notation used, that is $R_{j, l}$ vs. $\tilde{R}_{j, l}$ reflects the distinction between rules and associations.
$R_{j \mu}:$ if $e(t)$ is $A_{j}$ and $d e(t)$ is $B_{t}$ then $u(t)$ is $C_{j,}$ $R_{j+1, s}:$ if $e(t)$ is $A_{j+1}$ and $\operatorname{de}(t)$ is $B_{t}$ then $u(t)$ is $C_{j+1, t}$ $R_{j+L+1}:$ if $e(t)$ is $A_{j+1}$ and $\operatorname{de}(t)$ is $B_{l+1}$ then $u(t)$ is $C_{j+L+1}$ $R_{,, 1+1}:$ if $e(t)$ is $A_{j}$ and $d e(t)$ is $B_{t, 1}$ then $u(t)$ is $C_{j,+1}$ with each rule satisfied to some degree. The corresponding truth value is defined, for instance for the first rule, by

$$
\mu_{j, d}=\min \left(\mu_{i_{j}}(e(t)), \mu_{i_{1}}(\operatorname{de}(t))\right)
$$

or, alternatively by

$$
\mu_{j, l}=\mu_{i_{j}}(e(t)) \cdot \mu_{i_{1}}(\operatorname{de}(t))
$$

The truth values of other rules in the above set are similarly defined.

Note that the product instead of min results in interactivity between the truth values of the components of the antecedent clause. This fact is essential to our analytic treatment(Langari and Tomizuka 1990.)

Now, representing the consequent clause of each $R_{j,}$ rule, that is , by its single representative, or defuzzified, value that is $U_{j, l}$, defined as

$$
U_{j, l}=\frac{\int u \mu_{\tilde{c}_{j l}}(u)}{\int \mu_{\tilde{c}_{\jmath}}(u)}
$$

the control action, $u(t)$, is computed as:

$$
u(t)=\frac{\sum_{j, l} \mu_{,, l} U_{, t}}{\sum_{, l} \mu_{j, l}}
$$

where $j$ and $l$ range over the indices of all applicable rules. Note that this approach is based on a variation of the Centroid of Area(COA) defuzzification rule(Zimmermann 1991), but has improved analytical properties(Langari and Tomizuka 1990).

\section{ANALYSIS OF FUZZY LOGIC CONTROL ALGORITHMS.}

Consider the single input, single output fuzzy linguistic control system shown in Figure 1. Here we develop an analytic description of the control law in the form, $u=F L C(e, d e)$. 


\section{Definitions and Assumptions}

Let us denote the domains of definition of $e$, de, and $u$ by $E, D E$, and $U$ respectively. Then, as shown in Figure 2, collections $\dot{\alpha}=\left\{\tilde{A}_{j}\right\}$, $9 \tilde{B}=\left\{\tilde{B}_{l}\right\}$, and $\tilde{\nabla}=\left\{\tilde{C}_{J, 1}\right\}$ of unimodal, convex, and normal fuzzy subsets(Dubois and Prade 1980) effectively partition $E, D E$, and $U$, respectively, as follows.

Each element $\dot{A}$, of $\dot{A}$ is centered at some $E, \in E$ and is further characterized by a pair $L,(\cdot)$ and $R_{j}(\cdot)$ of left and right characteristic functions(cf. Appendix A). Similarly, each $\bar{B}_{1} \in \overline{B^{\circ}}$ is centered at some $D E_{1} \in D E$ and is characterized by $L_{i}^{\prime}(\cdot)$ and $R_{l}^{\prime}(\cdot)$. Moreover, each element $\tilde{C}_{j,}$ is represented by its defuzzified value, $U_{j, l}$.

We further place some constraints on $\bar{\alpha}$ and $\bar{g}$ as follows. First, we require that $\bar{\alpha}$ and $\bar{s}$ form true fuzzy partitions of $E$ and $D E$ respectively.

Assumption 1. Let $\dot{\alpha}=\left\{\tilde{A}_{i}\right\}$ (and $\bar{B}=\left\{\tilde{B}_{i}\right\}$ ) be collection(s) of fuzzy subsets defined over $E$ (and $D E$.) Then. for each element $e \in E$

$$
\sum_{j} \mu_{i_{j}}(e)=1
$$

\section{(A similar condition holds for $\boldsymbol{B B}^{\circ}$ )}

The interpretation of Assumption 1 is that, externally, fuzzy classification must be compatible with feature based classification in terms of classical sets, where each element is categorized under one and only one class. This assumption is crucial to the development of our results and in effect amounts to objectification of the control law.

A sufficient condition for Assumption 1 to hold is that the characteristic functions of $\tilde{A}_{j}$ (and $\tilde{B}_{l}$ ) be linear ${ }^{2}$ :

Assumption 2. For each $j$, let $\tilde{A}_{j} \in \tilde{A}$ be defined in terms of a pair $L_{j}(\cdot)$ and $R_{j}($.$) of left and right$ characteristic functions. Then

\footnotetext{
${ }^{2}$ A generalization of this condition, where nonlinear characteristic functions are allowable, is possible. The present discussion. however, does not hinge on this fact. The interested reader may refer to Langari(1992).
}

$$
\begin{aligned}
& R_{j}(e)=1-\left(e-E_{\jmath}\right) / \beta_{j} \\
& L_{j}(e)=1-\left(E_{j}-e\right) / \alpha_{j}
\end{aligned}
$$

and given $j$, and $j+1$, the line segments defined by $R_{j}(\cdot)$ and $L_{\jmath+1}(\cdot)$ intersect $E$ precisely $a t E$, and $E_{j, 1}$ respectively. (A similar condition holds for gi.)

This assumption implies that, as shown in Figure 3,
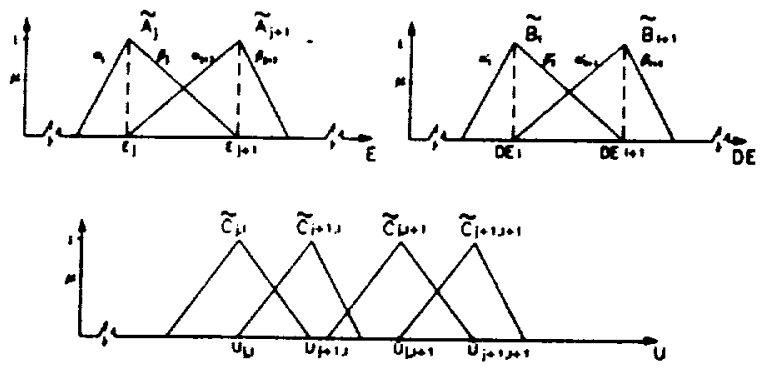

Figure 3. True Fuzzy Partitioning.

$\beta$, and $\alpha_{j+1}$, respectively representing the inverse of the slopes of the line segments defined by $R,(\cdot)$ and $L_{\mu, 1}($.$) , must be equal. Let us denote this$ unique slope by $m_{j}$ :

$$
m_{j}:=\frac{1}{\beta_{j}}=\frac{1}{\alpha_{j, 1}} .
$$

Similarly, $\alpha_{t, 1}^{\prime}$ and $\beta_{l}^{\prime}$, must also be equal; let us define $m_{i}^{\prime}:=\frac{1}{\alpha_{1+1}^{\prime}}=\frac{1}{\beta_{i}^{\prime}}$ to clearly indicate this fact as well. Consequently, we can define $\Delta E$, and $\Delta D E_{\text {, }}$ as follows:

$$
\begin{aligned}
& \Delta E_{\jmath}=E_{\jmath .1}-E_{\jmath}, \\
& \Delta D E_{l}=D E_{l+1}-D E_{l} .
\end{aligned}
$$

Let us also define $K_{, l}$ and $K_{j, l}^{\prime}$ as follows.

Definition 1. Let us denote the functional relationship between $U_{j,}, E_{j}$ and $E_{j, 1}$ as:

$$
U_{\lambda, l}=K_{, l} E_{j}+K_{j, l}^{\prime} D E_{l}
$$

Then for each pair, $j$ and $l, K_{j, l}$ and $K_{j, l}^{\prime}$ are implicitly defined by (II). 
Note that (11) simply relates $U_{J, l}$ to $E_{j}$ and $D E_{l}$ in a compact form and does not in any way constrain $U_{j, l}$.

We further define $\Delta K^{j}{ }_{j \cdot 1,1 \ldots \text { as follows: }}$

$$
\begin{aligned}
& \Delta K_{j+1, l}^{\prime}=K_{j+1, l}-K_{j, l}, \\
& \Delta K_{j, l+1}^{\prime}=K_{j, l+1}-K_{j, l}, \\
& \Delta K_{j+1, l+1}=K_{j+1, l+1}-K_{j, l} . \\
& \Delta K^{\prime \prime}{ }_{j, l+1}=K_{j, l+1}^{\prime}-K_{j, l}^{\prime}, \\
& \Delta K^{\prime \prime}{ }_{j+1, l}=K_{j \cdot 1, l}^{\prime}-K_{j,}^{\prime}, \\
& \Delta K_{j+1, l+1}^{\prime}=K_{j+1, l+1}^{\prime}-K_{j, l}^{\prime} .
\end{aligned}
$$

Now in view of the above assumptions the expression for $u(t)$, given by (4), resolves into

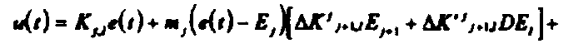

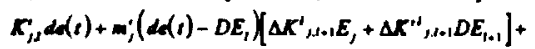

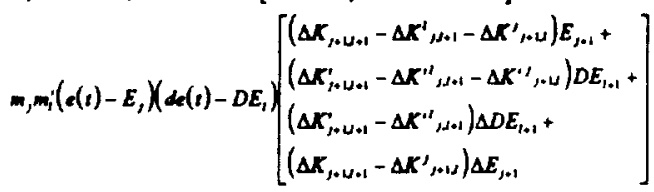

The implication of the above formulation is that a given fuzzy logic control algorithm in effect amounts to a nonlinear control law that is further described in terms of three terms: one that is linear in each of $e(t)$ and $d e(t)$, one that is linear in each of $e(t)-E_{j}$ and $d e(t)-D E_{l}$, and finally one that is bilinear in the latter two terms. In effect the control law given by (18) reflects the capacity of fuzzy logic control to interpolate across the situations where individual control rules are directly applicable. We will see next how this capacity can be used to develop a control strategy that deals effectively with nonlinearities that commonly occur in process control.

\section{APPLICATION}

Let us consider the dynamic system:

$$
\begin{aligned}
\dot{x}_{1} & =a_{1} x_{1}+a_{2} x_{2}+b u, \\
\dot{x}_{2} & =x_{1}, \\
y & =x_{2}-x_{1},
\end{aligned}
$$

which reflects the behavior of a rather broad class of industrial processes; it is a relatively low order model, and has the somewhat dubious distinction of being non-minimum phase. The parameters, $a_{1}$, and $a_{2}$ are given by

$$
\begin{aligned}
& a_{1}=a_{10}+\delta a_{1}, \\
& a_{2}=a_{20}+\delta a_{2},
\end{aligned}
$$

where $\delta a_{1}$ and $\delta a_{2}$ reflect the variations in the plant parameters.

Suppose now, as it is commonly done in practice, we knew the process model and were to design a simple proportional plus integral control law:

$$
u=k_{p} e+k_{i} \int_{0}^{1} e d \tau,
$$

perhaps based on nominal values of the plant parameters, $a_{10}$, and $a_{20}$, as follows.

The plant and controller transfer functions are given by:

$$
\begin{aligned}
& G_{p}(s)=\frac{1-s}{s^{2}+a_{10} s+a_{20}}, \\
& G_{c}(s)=\frac{k_{c}(s+\gamma)}{s},
\end{aligned}
$$

where $\gamma>0, k_{c}=k_{p}$ is same as the proportional control gain, and $k_{i}=\gamma k_{c}$ is the equivalent integral gain.

Now, assuming that the closed loop system will behave as a dominantly second order system, the closed loop characteristic equation is given by

$$
A(s)=(s+p)\left(s^{2}+2 \xi \omega s+\omega^{2}\right),
$$

where $p$ is assumed large, we can use any number of ways of selecting $\xi$ and $\omega-$ and thus $k_{c}$ and $\gamma$ - (Franklin, Powell, and Emami-Naeini 1991). For instance, we can simply pre-select $\gamma$ and then choose $\xi$ for desired response pattern and thus determine the gain $k_{c}$.

In practice, however, variations in the parameters of the plant, that is $\delta a_{1}$ and $\delta a_{2}$, affect the behavior of the process, and as a result the desired response is not reproduced as predicted. For instance, let us 
suppose that these variations are function of the process error $^{3}, e$ :

$$
\begin{aligned}
& \delta a_{1}=-\alpha \operatorname{sgn}(\mathrm{e}), \\
& \delta a_{2}=-\alpha \operatorname{sgn}(\mathrm{e}),
\end{aligned}
$$

where $\alpha>0$.

This situation happens in arc welding, for instance, where active heating and only passive cooling is available(Langari and Tomizuka 1988), A consequence of this change is that a fixed set of gains will not work well, no matter what values one chooses. Alternatively, one may resort to adaptive control. Generally, however, this approach requires slow variation in the plant parameters. One could also, in principle, rely on robust control, perhaps within the $H_{\text {- }}$ framework. The drawback of this approach, however, is that while robust performance may be guaranteed, uniformly robust performance is not. These claims should not be surprising since neither adaptive control or robust control is really meant to compensate for strong nonlinearities in the plant model.

Given this fact, therefore, one should at least ideally consider nonlinear control- global or feedback linearization. Indeed if the nature and extent of nonlinearity is known reasonably well, through a reasonably accurate plant model, one would do just that. Moreover, even in the absence of a formal model, it is our conjecture that the human operator of the process, having learned the peculiarity of its behavior, develops response behavior that in practice amounts to a nonlinear control scheme that compensates for the dominantly nonlinear, and undesired, characteristics of the process. In effect s/he globally linearize the process and compensates for the deficiencies in its dynamic response characteristics.

In the context of the current example, in particular, it seems plausible that a human operator would be able to compensate for variations in the plant parameters, as required and as shown in Figure 4 produce response pattern superior to any linear control strategy.

\section{Analysis of Response Pattern}

Clearly, assuming that the control action of the human operator is described in linguistic form, the key factor would be the manner of definition of the rule set and its constitutive linguistic term set. This is evident, as shown in Figure 5, in the manner of

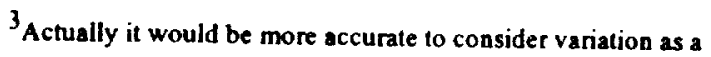
function of the process input so as to reflect the coupling between state and input variables. however, in closed loop control the input is itself a function of the error.
}

definition of the linguistic term set defined over the domain of definition of the process error.

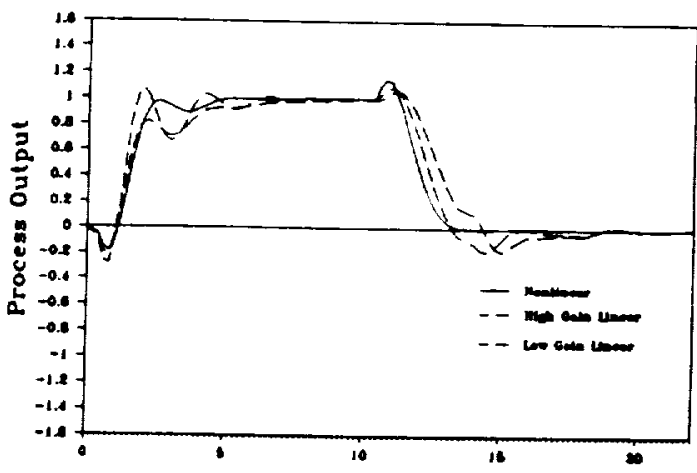

Figure 4. Response patterns of fuzzy vs. linear control.
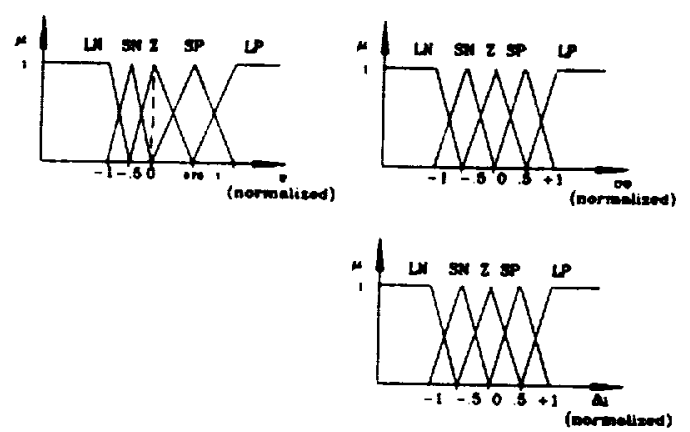

Figure 5. Definition of fuzzy membership functions.

In particular, the asymmetry in the definition of terms such as small-positive and small-negative, denoted in the figure by SP and SN respectively, reflects the variation in the proportional gain across the origin of the domain of definition of $e$.

Now, using the formalism presented earlier, one can show that the operator's action, interpreted above in linguistic terms, effectively amounts to a nonlinear control scheme

$$
u=k_{p} e+k_{i} \int_{0}^{1} e d \tau
$$

where $k_{p}$, is given by $k_{p}=k_{p 0}-\alpha \operatorname{sgn}(e) / b$, which in the case of the regulation problem, in effect cancels the nonlinear terms which we attributed earlier to parametric variation ${ }^{4}$.

\section{CONCLUSION}

\footnotetext{
${ }^{4}$ In reality when the setpoint is changed, this cancellation does not hold in the exact sense, however, since the plant dynamics is still linearized and stable, treating the setpoint change effect as a disturbance which results in diminishing transients is a reasonable assumption.
} 
In this paper we showed how fuzzy control can be viewed as a paradigm for designing nonlinear control strategies in situations where the plant model is not a priori known- at least sufficiently well- to warrant the application of conventional control theory. In particular, we made a point regarding the use of fuzzy control in situations that occur frequently in industrial process control where (nonlinear)dependence of the parameters of the plant on its state variables precludes the application of linear control theory and thus nonlinear control, albeit by means of fuzzy control, seems to be the most appropriate approach. The framework presented here, however, is somewhat restrictive in that it requires a specific form for parametrization of fuzzy sets $(L R)$ and places some restrictions on the manner of definition of the control rules $\left(\sum \mu=1\right)$. To be more widely applicable, this framework needs to to allow for a wider range of nonlinear control schemes and also to allow for nonparametrized fuzzy sets.

\section{APPENDIX.}

\section{A. Parametrization}

Although not absolutely essential, parametrization simplifies quantitative description of fuzzy subsets. In $L R$ parametrization(Dubois and Prade 1980), a fuzzy subset $\bar{A}$, defined on some universe of discourse $U$, is characterized, in terms of its membership function, as follows:

$\mu_{\lambda}(u)= \begin{cases}L\left(\left(u_{0}-u\right) / \alpha\right) & \text { if } u \leq u_{0} \\ R\left(\left(u-u_{0}\right) / \beta\right) & \text { if } u>u_{0}\end{cases}$

where, as shown in Figure $6, L(\cdot)$ and $R($. characterize the left and right halves of $\tilde{A}$, relative to its center value, $u_{0}$, that is where the linguistic term that $\tilde{A}$ represents fully achieves its meaning, or is maximally satisfied. Moreover, $\alpha$ (and $\beta$ ) parametrize $L(\cdot)$ (and $R(\cdot)$ ), which typically takes the form

$$
L(x)=\left\{\begin{array}{l}
\max \left(0,1-|x|^{p}\right), \text { or } \\
\mathrm{e}^{-|x|^{\prime}}, \text { or } \\
\frac{1}{1+|x|^{p}}
\end{array},\right.
$$

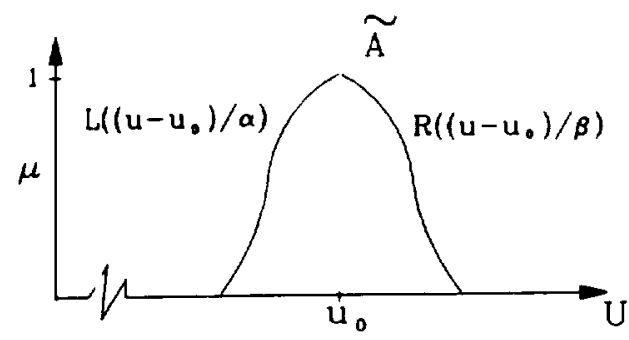

Figure 6. Parametrization of a fuzzy subset.

Finally, it is sometimes sufficient to use a simple linear form, based on $L(x)=\max (0,1-|x|)$, in which case, $\alpha$ (or $\beta$ ), discussed above, would represent the inverse of the slope of the characteristic function:

$$
\begin{aligned}
& R(u)=1-\left(u-u_{0}\right) / \beta, \\
& L(u)=1-\left(u_{0}-u\right) / \alpha .
\end{aligned}
$$

\section{REFERENCES}

Dubois, D and Prade, H.(1980). Fuzzy Sets and Systems. Academic Press, NY.

Franklin G., Powell, D., and Emami-Naeini, A.(1991). Feedback Control of Dynamic Systems. Second Edition. Addison Wesley, NY.

Langari, G.(1990) A Framework for Analysis and Synthesis of Fuzzy Linguistic Control Systems. Ph.D. Dissertation. University of California, Berkeley.

Langari G.(1992) Nonlinear Formulation of a Class of Fuzzy Linguistic Control Systems. In Proceedings of the American Control Conference.

Langari G. and Tomizuka, M.(1988). Fuzzy Linguistic Control of Arc Welding. In Sensors and Controls for Manufacturing: Proceedings of the ASME Winter Annual Meeting. Vol. PED-33. ASME, NY.

Langari, G. and Tomizuka, M.(1990) Analysis and Synthesis of Fuzzy Linguistic Control Systems. In R. Shoureshi(Ed.) Intelligent Control 1990: Proceedings of the ASME Winter Annual Meeting. Vol. DSC-23. ASME, NY.

Mamdani, E. and Assilian, S.(1975). An Experiment in Linguistic Synthesis with a Fuzzy Logic Controller. International Journal of Man Machine Studies. Vol. 7, No. 1.

Sugeno, M.(1985). Industrial Applications of Fuzzy Control. North Holland, Amsterdam.

Zimmermann, H.(1991) Fuzzy Set Theory and its Applications. Second Edition Kluwer Academic Publishers. 